\title{
Modeling Crosstalk Noise for Deep Submicron Verification Tools
}

\author{
Pirouz Bazargan Sabet ${ }^{\S}$ \\ ${ }^{\S}$ LIP6 - University of Paris 6 \\ 4 Place Jussieu - 75005 Paris - France \\ Pirouz.Bazargan-Sabet@lip6.fr
}

\author{
Fabrice Ilponse ${ }^{\S \dagger}$ \\ ${ }^{\dagger}$ ST-Microelectronics \\ 850 Rue Jean Monnet - 38926 Crolles - France \\ Fabrice.Ilponse@lip6.fr
}

\begin{abstract}
$^{1}$
In deep submicron technologies, the verification task has to cover some new issues to certify the correctness of a design. The noise produced by crosstalk couplings is one of these emerging problems. In this paper, we propose a model to evaluate the peak value of the noise injected on a signal when its neighboring signals make their transitions. This model has been used in a prototype verification tool and has shown a satisfying performaceaccuracy ratio.
\end{abstract}

\section{Introduction}

In deep submicron processes, designers have to face new problems that have been somewhat neglected until now. The noise introduced by the crosstalk coupling is one of these emerging issues that may cause timing and, in some extreme cases, functional failures in the circuit. Some efforts have been initiated to include crosstalk analysis in design methodologies [1][2][3][4][5][6]. Design tools, such as routers, must be modified [7][8] to take into account and to reduce the effect of the crosstalk. Also, new verification tools must be provided to certify the correctness of a design against crosstalk misfunction [9].

In this paper, we propose a simple and still accurate model to evaluate the importance of the crosstalk noise for each signal of the circuit. This model has been developed to be incorporated within a crosstalk noise verification tool. The next section describes the crosstalk phenomenon. Section 3 details our crosstalk noise evaluation model. The background of this study is exposed in section 4. Some results are shown in section 5, comparing the noise obtained from the proposed model against a SPICE simulation. Concluding remarks and future works are depicted in the last section.

\footnotetext{
${ }^{1}$ This study is part of a Ph.D. thesis currently undergoing at University of Paris 6 and funded by ST-Microelectronics.
}

\section{The Crosstalk Noise}

The crosstalk phenomenon is due to the existence of a capacitance between two neighboring wires. Whenever a wire makes a transition, a noise is produced through this coupling capacitance on the other wire of the couple. Let's consider two signals $A$ and $V$ driven by two inverters (Fig. 1). When the signal $A$ makes a transition, a noise is injected on the signal $V$. If $V$ is in a steady state, the noise has the form of a spike and is absorbed by the $V$ 's driver after some delay. On the contrary, if $V$ is making its own transition in the same time, the crosstalk noise leads in a shorter or longer transition delay. The signal $A$ is called the aggressor and $V$ the victim.

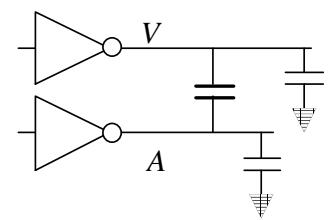

Fig 1 : Two signals in crosstalk coupling

Many factors contribute to the apparition of the crosstalk noise in submicron processes: the shape of wires, the reduction of the distance between wires, the greater number of metal layers, etc. These factors tend either to reduce the capacitance to the ground or, to increase the coupling capacitance. Another parameter that determines the importance of the noise is the impedance of the aggressor and victim's drivers.

In this paper, we propose a model to calculate the peak value of the noise when the victim is in a steady state. The same model may be used to estimate the modification of the victim's transition delay.

\section{Crosstalk Noise Evaluation Model}

In a real circuit, a given signal may be coupled with several thousand signals, each of them having many other couplings. An analog simulation can provide a precise knowledge of the impact of transitions inside 
such a coupled system. However, its is well known that making such a simulation is unrealistic even for a hundred thousands transistor circuit.

To describe our model we propose to proceed by a three-step approach. In a first step, the influence of a single aggressor on a single victim is studied. Then, this model is extended to multiple aggressors. Finally, the real case of several aggressors producing a noise on several victims is pictured.

A first approximation consists of replacing signal's drivers by a simple resistance. The relevance of this replacement is discussed in a later section.

\subsection{Single Aggressor - Single Victim}

Let consider the simple case of Fig. 2.

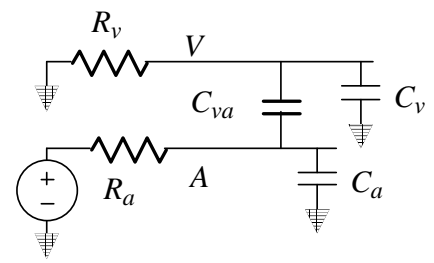

Fig 2: Single aggressor, single victim

Signal $V$ is supposed to be at the steady state $0\left(\mathrm{~V}_{\mathrm{ss}}\right)$. Signal $A$ makes a transition from 0 to $\mathrm{V}_{\mathrm{dd}}$. The waveforms of $A$ and $V$ can be obtained analytically by resolving the following system of two first order linear differential equations:

$$
\left\{\begin{array}{c}
v(t)+\tau_{v} v^{\prime}(t)-\alpha_{v a} a^{\prime}(t)=0 \\
a(t)+\tau_{a} a^{\prime}(t)-\alpha_{a v} v^{\prime}(t)=V_{d d}
\end{array}\right.
$$

where: $\tau_{v}=R_{v}\left(C_{v}+C_{v a}\right) \quad \alpha_{v a}=R_{v} C_{v a}$

$$
\tau_{a}=R_{a}\left(C_{a}+C_{v a}\right) \quad \alpha_{a v}=R_{a} C_{v a}
$$

Both $a(t)$ and $v(t)$ are a sum of two exponential terms.

$$
\begin{aligned}
& v(t)=K_{v}+L_{v} e^{-t / \tau_{1}}+M_{v} e^{-t / \tau_{2}} \\
& a(t)=K_{a}+L_{a} e^{-t / \tau_{1}}+M_{a} e^{-t / \tau_{2}}
\end{aligned}
$$

The system can be resolved after replacing $a(t)$ and $v(t)$ by their expression. $\tau_{1}$ and $\tau_{2}$ are the two roots of a quadratic equation:

$$
\begin{gathered}
\tau^{2}-\tau\left(\tau_{v}+\tau_{a}\right)+\tau_{v} \tau_{a}-\alpha_{v a} \alpha_{a v}=0 \\
\tau_{1}=\frac{1}{2}\left(\tau_{a}+\tau_{v}+\sqrt{\left(\tau_{a}-\tau_{v}\right)^{2}+4 \alpha_{v a} \alpha_{a v}}\right. \\
\tau_{2}=\frac{1}{2}\left(\tau_{a}+\tau_{v}-\sqrt{\left(\tau_{a}-\tau_{v}\right)^{2}+4 \alpha_{v a} \alpha_{a v}}\right.
\end{gathered}
$$

Then, the expression of $a(t)$ and $v(t)$ can be established:

$$
\begin{aligned}
& v(t)=V_{d d} \frac{\alpha_{v a}}{\tau_{1}-\tau_{2}}\left(e^{-t / \tau_{1}}-e^{-t / \tau_{2}}\right) \\
& a(t)=V_{d d}\left(\frac{\tau_{v}-\tau_{1}}{\tau_{1}-\tau_{2}} e^{-t / \tau_{1}}+\frac{\tau_{v}-\tau_{2}}{\tau_{2}-\tau_{1}} e^{-t / \tau_{2}}+1\right)
\end{aligned}
$$

The expression of $v(t)$ can be used to obtain the peak time and then the peak value.

$$
\begin{aligned}
t_{\text {peak }} & =\frac{\tau_{1} \tau_{2}}{\tau_{1}-\tau_{2}} \log \left(\frac{\tau_{1}}{\tau_{2}}\right) \\
v_{\text {peak }} & =V_{d d} \alpha_{v} \tau_{1}{ }^{\left(\frac{\tau_{1}}{\tau_{2-}}\right)} \tau_{2}{ }^{\left(\frac{\tau_{2}}{\tau_{1-} \tau_{2}}\right)}
\end{aligned}
$$

\subsection{Multiple Aggressors - Single Victim}

The above situation can be extended to a more general case where a signal $V$ is aggressed by several aggressors $A_{l}, \ldots, A_{n-l}$ assuming that all these aggressors make their transition at the same time and in the same direction (the worst case). In a similar way, a system of $n$ linear first order differential equations can be expressed:

$$
\left\{\begin{array}{l}
v(t)+\tau_{v} v^{\prime}(t)-\sum_{i=1}^{n-1} \alpha_{v a_{i}} a_{i}{ }^{\prime}(t)=0 \\
\vdots \\
a_{i}(t)+\tau_{a_{i}} a_{i}{ }^{\prime}(t)-\alpha_{a_{i} v} v^{\prime}(t)=V_{d d}
\end{array}\right.
$$

Therefore, $v(t)$ is a sum of $\mathrm{n}$ exponential terms. The resolution of this system requires the calculation of the $n$ roots of an n-degree equation. Clearly, this calculation cannot be performed analytically. Even if an iterative algorithm may be used to determine these $n$ roots, we believe that the complexity of such a resolution is intractable for a life size circuit with millions of signals. A more efficient way is to transform the system such as the contribution of each aggressor could be calculated separately and summed with the other aggressors' effect. In our approach, we propose to substitute each aggressor by a current source with a given behavior.

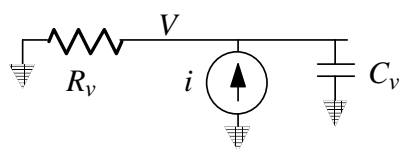

Fig 3: A source current as an aggressor

Let's consider the case of Fig. 3 where the aggressor has been replaced by a current source. Assuming that the behavior of the source is

$$
i=I_{0} e^{-t / \tau_{i}}
$$

the expression of the victim would be a sum of two exponential terms as in the previous sub-section.

$$
v(t)=R_{v} I_{0} \frac{\tau_{i}}{\tau_{v}-\tau_{i}}\left(e^{-t / \tau_{v}}-e^{-t / \tau_{i}}\right)
$$


Then, the two parameters of the current source can be set such as the peak time and the peak value remain the same as previously.

$$
\begin{aligned}
& \frac{\tau_{v} \tau_{i}}{\tau_{v}-\tau_{i}} \log \left(\frac{\tau_{v}}{\tau_{i}}\right)=\frac{\tau_{1} \tau_{2}}{\tau_{1}-\tau_{2}} \log \left(\frac{\tau_{1}}{\tau_{2}}\right) \\
& I_{0}=\frac{V_{d d}}{R_{v}} \frac{\alpha_{v a}}{\tau_{1}}\left(\frac{\tau_{2}}{\tau_{1}}\right)^{\left(\frac{\tau_{2}}{\tau_{1}-\tau_{2}}\right)}\left(\frac{\tau_{v}}{\tau_{i}}\right)^{\left(\frac{\tau_{v}}{\tau_{v}-\tau_{i}}\right)}
\end{aligned}
$$

The first equation is solved by linearization to determine $\tau_{i}$. Then, $I_{0}$ is calculated from the second equation.

Applying this approach, an equivalent current source is attributed to each aggressor and its parameters are fixed. When a given aggressor is involved in a crosstalk coupling, the equivalent current source is called instead.

As a result, a system equivalent to the Multiple aggressors-Single victim case is built.

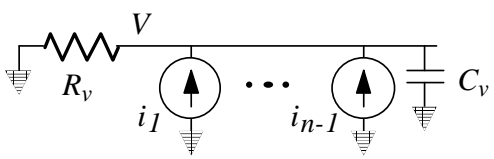

and the expression of $v(t)$ is assessed

$$
v(t)=R_{v} \sum_{k=1}^{n-1} I_{0_{k}} \frac{\tau_{i_{k}}}{\tau_{v}-\tau_{i_{k}}}\left(e^{-t / \tau_{v}}-e^{-t / \tau_{i_{k}}}\right)
$$

The peak time of the noise is then given by:

$$
\sum_{k=1}^{n-1} I_{0_{k}} \frac{\tau_{i_{k}}}{\tau_{v}-\tau_{i_{k}}}\left(\frac{1}{\tau_{v}} e^{-t_{\text {peak }} / \tau_{v}}-\frac{1}{\tau_{i_{k}}} e^{-t_{\text {peak }} / \tau_{i_{k}}}\right)=0
$$

Once again, this equation can be resolved by linearization. Then the peak value is evaluated.

It is important to notice that the substitution of an aggressor by its equivalent current source represents an approximation that neglects the effect of an aggressor on other aggressors through the victim. Nevertheless, this impact is a second order effect and can actually be neglected.

\subsection{Multiple Aggressors - Multiple Victims}

In a real circuit, crosstalk interactions affect signals in a more complex way. A given signal is coupled to many aggressors. Some of these are making a rising transition, some others a falling. The influence of these aggressions are added and a rising transition may compensate the effect of a falling one. The above equivalent model is sensitive to the direction of transitions and reflects this compensation.

However, not all aggressors are active at each time. Silent aggressors do not participate to the noise produced on the victim. On the contrary, their coupling capacitance can be seen as a part of the capacitance to the ground and contributes to the stability of the victim.
On the other hand, the transition of a signal has an effect on all the neighboring signals that are in crosstalk coupling with this one. In other terms, a given aggressor has several victims and the noise produced by its transition is spread on all the victims. Thus, the existence of other victims contributes to the reduction of the noise on a given victim.

The way these two last phenomena are taken into account is described in this sub-section.

Let's consider once again the case of Fig 2, this time, from the aggressor's point of view. The victim, through the coupling capacitance $C_{v a}$, produces a perturbation on $A$ during its transition. As discussed before, in the presence of the victim, $a(t)$ is no more a simple exponential but the sum of two exponential terms. More precisely, the perturbation is caused by the resistance $R_{v}$ and the current that it may drive.

Let's examine the two extreme situations: $R_{v} \rightarrow 0$ and $R_{v} \rightarrow \infty$. When $R_{v} \rightarrow 0$, the victim is equivalent to $C_{v a}$ connected to the ground and

$$
a(t) \rightarrow V_{d d}\left(1-e^{-t / \tau_{1}}\right)
$$

When $R_{v} \rightarrow \infty$, the victim is equivalent to a capacitance connected to the ground :

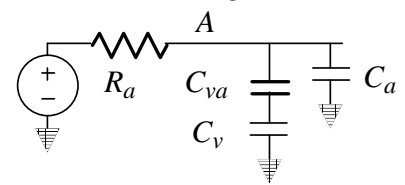

The effect of the victim on the aggressor can be seen as an additional delay during the transition of this one. Thus, the victim can be approximated by an equivalent capacitance, $C_{e q}$, connected to the ground.

$$
\frac{C_{v a} C_{v}}{C_{v a}+C_{v}}<C_{e q}<C_{v a}
$$

Determining $C_{e q}$ consists in characterizing a simple exponential $a_{e q}(t)$ that represents the best approximation of $a(t)$.

$$
a_{e q}(t)=V_{d d}\left(1-e^{-t / \tau_{e q}}\right) \text { where } \tau_{e q}=R_{a}\left(C_{a}+C_{e q}\right)
$$

We propose to use the quadratic difference as the minimization criteria.

$$
D\left(\tau_{e q}\right)=\int_{0}^{\infty}\left(a_{e q}(t)-a(t)\right)^{2} d t
$$

The minimum distance is given by $\frac{d D\left(\tau_{e q}\right)}{d \tau_{e q}}=0$ which leads to a fourth degree equation. Determining $C_{e q}$ requires to identify one of the 4 roots of the above equation. Once again we use a successive linearization method to perform this calculation. 
Put all together, our crosstalk noise model can be summarized as follows:

1. For each victim, replace silent aggressors by an equivalent capacitance.

2. Replace the other victims of the remaining active aggressors, by an equivalent capacitance.

3. Replace each active aggressor by an equivalent current source and determine the peak noise.

\section{Background}

Modeling a transistor as a simple resistance could seem unrealistic for deep submicron processes.

Actually, our crosstalk noise model is based on the same transistor model as the one proposed by A. Hajjar in the static timing analysis tool, TAS. This model, called MCC, is particularly adapted to short channel MOS transistors. Here we give a brief overview of this model. A detailed description of MCC can be found in [10][11]. In MCC, a MOS transistor and the current it can drive are characterized by the following expressions:

$$
\begin{array}{ll}
\text { Blocked mode }\left(V_{g s}<V_{t}\right) & I_{d s}=0 \\
\text { Linear mode }\left(V_{d s}<V_{s a t}\right) & I_{d s}=\frac{V_{d s} I_{s a t}}{V_{s a t}} \\
\text { Saturated mode }\left(V_{d s}>V_{s a t}\right) & I_{s a t}=\frac{W A\left(V_{g s}-V_{t}\right)^{2}}{L\left(1+B\left(V_{g s}-V_{t}\right)\right)} \\
\text { Saturation tension } & V_{s a t}=K\left(V_{g s}-V_{t}\right)
\end{array}
$$

$A, B$ and $K$ are constant parameters that depend on the integration process. In TAS, the transition of a signal is modeled using a hyperbolic tangent form:

$$
v(t)=V_{t}+\left(V_{\text {end }}-V_{t}\right) \operatorname{Th}\left(\frac{t-t_{0}}{F}\right)
$$

$t_{0}$ and $F$ characterize the transition time and slope.

Let consider an inverter loaded by a capacitance. Given that the N-transistor's grid makes its transition following the above form, the expression of the drain can be determined, assuming that the transistor is always in the saturation region. Then, supposing that the drain's waveform obeys also to the hyperbolic tangent form, the two parameters of the transition can be determined. This calculation is performed such as the transition time and slope match the time and the slope obtained from the transistor's expression at $\mathrm{V}_{\mathrm{dd}} / 2$.

The experience has shown that, using this model, TAS can reach a precision less than $10 \%$ compared to SPICE when evaluating the longest path of a circuit designed with up-to-date processes.

In our model, the hyperbolic tangent function that characterizes the transition of an aggressor is approximated by an exponential form. Then, the resistance equivalent to the aggressor's driver is obtained from the time constant of this exponential.

Unlike aggressors that make a complete transition, the driver of a victim is mostly in its linear region. Therefore, the resistance that represents the victim's driver is calculated directly from the transistor's expression, assuming that $\mathrm{V}_{\mathrm{GS}}$ remains stable.

\section{Results}

The proposed model has been specified as part of a project that aims the development of a crosstalk noise evaluation tool. Fig. 4 illustrates a simplified flow chart of the prototype tool.

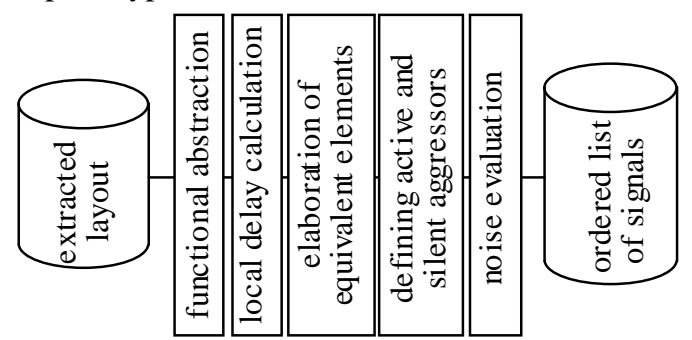

Fig 4: Simplified flow chart

The input is an extracted layout that includes parasitic capacitances. A functional abstractor is used to convert the transistor netlist into a gate netlist. Then, a static timing analysis module is called to attribute a delay to each gate regardless of the crosstalk noises. A third module elaborates the equivalent elements. Two equivalent resistances are defined for each signal's driver, one as a victim and one as an aggressor. An equivalent capacitance and current source are also specified for the aggressor in each crosstalk coupling.

The next step consists in evaluating the time intervals inside which each signal is silent or active. This evaluation is performed through a symbolic event driven simulation on the gate netlist produced by the functional abstractor.

In the last step, the crosstalk noise induced on each signal is estimated with respect to the proposed model. Only those aggressors whose active time intervals have an intersection are taken as active. Then, the configuration that generates the highest peak is considered. The output of the tool is an ordered list of signals in regard of this peak value.

Fig. 5 and 6 give the example of a small circuit (Table 1) designed with a $0.25 \mu$ and 2 level metal layers. Fig. 5 shows the ordered list of signals and the corresponding peak value. It compares the value obtained from a SPICE simulation and the estimation given by the proposed model. Fig. 6 concerns the same circuit and presents the relative error of the estimated peaks compared to the SPICE simulation. 


\begin{tabular}{|l|c|c|}
\hline \multicolumn{2}{|c|}{ AMD2901-Control } \\
\hline Number of transistors & \multicolumn{2}{c|}{818} \\
\hline Number of gates & \multicolumn{2}{c|}{236} \\
\hline Avg and max aggressors/victim & 11 & 86 \\
\hline
\end{tabular}

Table 1: Amd2901

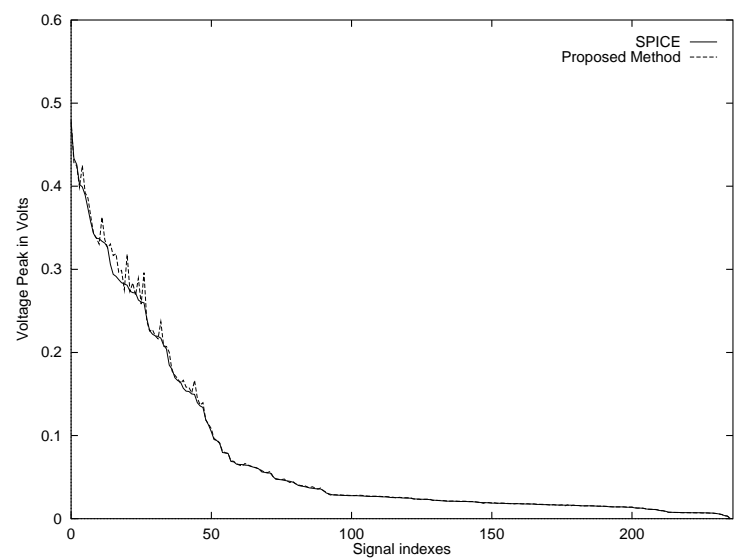

Fig 5: Peak value: proposed model vs. SPICE

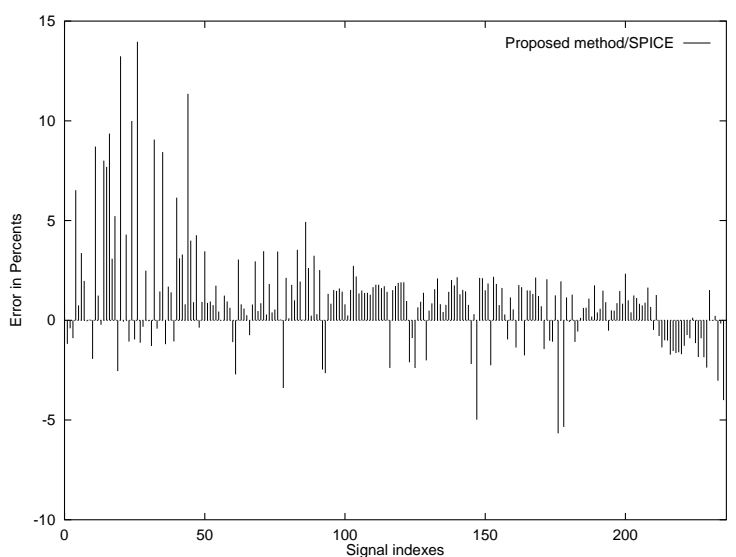

Fig 6: Relative error: proposed model vs. SPICE

The accuracy of the proposed model has been checked through other similar comparisons. The experience has shown that, compared to SPICE, our model leads to an error less than $15 \%$.

\begin{tabular}{|l|c|c|}
\hline \multicolumn{2}{|c|}{ RCUBE } \\
\hline Number of transistors & 275832 \\
\hline Number of gates & \multicolumn{2}{|c|}{73038} \\
\hline Avg and max aggressors/victim & 15 & 5465 \\
\hline
\end{tabular}

Table 2: Rcube

The computational cost of the method has been mesured on a Linux PC 500Mhz using a bigger circuit (Table 2) with 3 metal layers in a $0.25 \mu$ process. The peak computation step takes 164.6 seconds for 567578 peak computations (3450 computations/ sec).

\section{Conclusion}

A model to evaluate the peak value of the noise caused by crosstalk couplings on a signal has been presented. To reach a reasonable performance, several approximations have been made but, first order effects such as the presence of other victims and silent aggressors have been taken into account. The experience has shown that the proposed model represents a satisfying trade-off between performance and accuracy.

However, this model has to be extended to take into consideration some aspects that have been neglected. In deep submicron processes, interconnections contributes to the propagation delay of signals. A first development concerns the extension of the model to incorporate the resistance of interconnections.

The estimation of gates' delay is another aspect. In the presence of crosstalk noise the propagation delay of victims is changed. Thus, the crosstalk noise evaluation tool has to be coupled to the static timing analysis to rectify the gates' delay.

\section{References}

[1] R. Saleh, D. Overhauser, S. Taylor, "Full-Chip of UDSM Designs", IEEE/ACM, ICCAD, 1998, pp 453-460

[2] P. Chen, K. Keutzer, "True Crosstalk Noise Analysis", IEEE/ACM, DAC, 1999, pp 111-116

[3] K.L. Shepard, V. Narayanan, "Noise in Deep Submicron Digital Design", IEEE/ACM, ICCAD, 1996, pp 524-531

[4] B.N. Sheehan, "Predicting Coupled Noise in RC Circuits", IEEE, DATE, 2000, pp 517-521

[5] B. Choi, D.M. H. Walker, "Timing Analysis of Combinational Circuits Including Capacitive Coupling and Statistical Process Variation", IEEE, DATE, 2000, pp 49-54

[6] J.S. Yim, C.M. Kyung, "Reducing Cross-Coupling among Interconnect Wires in Deep Submicron Datapath Design”, IEEE/ACM, DAC, 1999, pp 485-491

[7] T. Xue, E.S. Kuh, Dongsheng Wang, "Post Global Routing Crosstalk Risk Estimation and reduction", IEEE/ACM, ICCAD, 1996, pp 302-309

[8] H. Zhou, D.F. Wong, "An Optimal Algorithm for River Routing with Crosstalk Contraints", IEEE/ACM, ICCAD, 1996, pp 310-315

[9] A. Odabasioglu, M. Celik, L.T. Pileggi, "PRIMA: Passive Reduced-order Interconnect Macromodeling Algorithm", IEEE/ACM, ICCAD, 1997

[10] A. Hajjar, "Modelisation des temps de propagation et analyse temporelle statique", Ph.D. dissertation, University of Paris 6, 1992

[11] A. Hajjar, R. Marbot, A. Greiner, P. Kiani, "TAS: An Accurate Timing Analyser for CMOS VLSI", EDAC, IEEE , 1991, pp 261-265 